\title{
Application of Human-Autonomy Teaming to an Advanced Ground Station for Reduced Crew Operations
}

\author{
Nhut Ho, Walter Johnson, Karanvir Panesar, Kenny \\ Wakeland, Garrett Sadler, Nathan Wilson, Bao \\ Nguyen \\ Human Automation Teaming Solutions, Inc. \\ Canoga Park, CA \\ nhut.ho@hats.solutions
}

\author{
Joel Lachter \\ Human Systems Integration Division \\ NASA Ames Research Center \\ Moffett Field, CA
}

\author{
Summer Brandt \\ San José State University \\ Moffett Field, CA
}

\begin{abstract}
Within human factors there is burgeoning interest in the "human-autonomy teaming" (HAT) concept as a way to address the challenges of interacting with complex, increasingly autonomous systems. The HAT concept comes out of an aspiration to interact with increasingly autonomous systems as a team member, rather than simply use automation as a tool. The authors, and others, have proposed core tenets for HAT that include bi-directional communication, automation and system transparency, and advanced coordination between human and automated teammates via predefined, dynamic task sequences known as "plays." It is believed that, with proper implementation, HAT should foster appropriate teamwork, thus increasing trust and reliance on the system, which in turn will reduce workload, increase situation awareness, and improve performance. To this end, HAT has been demonstrated and/or studied in multiple applications including search and rescue operations, healthcare and medicine, autonomous vehicles, photography, and aviation. The current paper presents one such effort to apply HAT. It details the design of a HAT agent, developed by Human Automation Teaming Solutions, Inc., to facilitate teamwork between the automation and the human operator of an advanced ground dispatch station. This dispatch station was developed to support a NASA project investigating a concept called Reduced Crew Operations (RCO); consequently, we have named the agent R-HATS. Part of the RCO concept involves a ground operator providing enhanced support to a large number of aircraft with a single pilot on the flight deck. When assisted by R-HATS, operators can monitor and support or manage a large number of aircraft and use plays to respond in real-time to complicated, workload-intensive events (e.g., an airport closure). A play is a plan that encapsulates goals, tasks, and a task allocation strategy appropriate for a particular situation. In the current implementation, when a play is initiated by a user, R-HATS determines what tasks need to be completed
\end{abstract}

and has the ability to autonomously execute them (e.g., determining diversion options and uplinking new routes to aircraft) when it is safe and appropriate. R-HATS has been designed to both support end users and researchers in RCO and HAT. Additionally, R-HATS and its underlying architecture were developed with generalizability in mind as a modular software applicable outside of RCO/aviation domains. This paper will also discuss future further development and testing of $R$ HATS.

Keywords-Automation; Human-Autonomy Teaming; Reduced Crew Operations; Ground Control Station

\section{INTRODUCTION}

Across virtually all industries, professionals are required to interact with increasingly sophisticated forms of automated systems for the performance of daily tasks. This ubiquity of automation is also not limited to the world of trained specialists on the job, but is more and more a part of people's daily lives due to the introduction of increasingly advanced automation in computers, tablets, cell phones, "smart" homes, and automobiles. NASA's Reduced Crew Operations (RCO) concept of operations, where operators of a ground control station (GCS) must provide concurrent supervision, management and assistance for multiple aircraft, relying on suites of automation to help provide a manageable and safe workload, is a case in point [1]. Here, human-autonomy teaming (HAT), a concept in which humans and automation dynamically interact through cooperation and teamwork, has been proposed as part of the solution to ensuring that RCO operators safely and appropriately trust in and rely on the 
complex automated systems involved in performing their jobs [2], [3].

In this paper, we describe key principles for successful human-autonomy teaming design. These principles include: situational awareness, automation transparency, bi-directional communication, operator-directed interfaces, and dynamic task allocation [4]-[6]. This discussion is then followed by an elaboration on a HAT agent designed for the RCO application. This agent, called R-HATS, integrates with an existing GCS and serves as a teamwork facilitator between RCO suites of automation and the human operator.

\section{DESIGN PRINCIPLES IN SUPPORT OF HAT}

\section{A. Provide Information that Matches Operator Required Situation Awareness}

Situation awareness (SA) is a critical concept for ensuring that an operator has sufficient knowledge and understanding of a situation for appropriate performance and decision making. In a seminal paper, Mica Endsley [7] advances a theory of situation awareness that spans multiple levels of information processing. Beyond a traditional focus solely on awareness of data, this theory presents a more advanced model encompassing information acquisition, processing, and projection into future states of events within the situation. To be situationally aware, Endsley argues, a person must have acquired SA across three levels: perception of elements in the environment (Level 1), comprehension of the current situation (Level 2), and projection of future status (Level 3).

\section{B. Provide Transparency to Facilitate Appropriate Trust Development}

The construct of transparency has been investigated and studied in the context of trust calibration by multiple researchers. Two design principles for transparency are of immediate interest. First, Lyons [8] proposed using the construct of transparency to guide the development of systems that facilitate appropriate operator trust in and reliance on automation. Lyons proposed that transparency produces shared awareness and shared intent between human operators and automation in two aspects: robot-to-human transparency and robot-of-human transparency. Robot-to-human transparency consists of the proper conveyance to the operator of the automation's understanding of the present situation. In other words: what is the automation doing, how is it doing that, and why? Conversely, robot-of-human transparency conveys to the operator what the automation knows about the human operator, such as the operator's preferences, tasks and goals, and current state (e.g., stress and fatigue).

Second, Chen and colleagues [9] developed the Situation Awareness-based Agent Transparency (SAT) model which provides guidelines for transparency that directly address Endsley's three levels of SA. Effectively, the three SAT levels provide transparency that allows an operator to answer three questions: What is the automation doing? (SAT Level 1), Why is the automation doing that? (SAT Level 2), and What should
I expect to happen next? (SAT Level 3). Unlike Endsley's model of SA, all three SAT levels are not always required for ideal interface design. Instead, the specific transparency levels employed should be considered on a case-by-case basis. Overall, the SAT model provides guidance for communicating to the user what the automation is doing and how ideal teaming between humans and automation occurs when different SAT transparency levels are achieved.

\section{Use Bi-Directional Communication to Facilitate HAT}

Bi-directional communication is an integral principle for crew resource management. On a conventional, two-person flight deck, it is standard procedure that the pilot flying and pilot monitoring are constantly communicating as the two parties work collaboratively to generate ideas, evaluate options and make best decisions, and find and correct mistakes, errors, or misunderstandings. A good HAT agent should mimic this form of interaction between human and automated team members [5]. This means that information for decisions regarding planning and execution of tasks is passed back and forth between team members. This would allow the operator to understand what the automation is doing and why, to work out proposed solutions to situations that may arise unexpectedly, and to communicate the potential outcomes of various courses of action. For its highly collaborative form of interaction and decision making, bi-directional communication is crucial for the improvement of teamwork between operator and automation.

For example, the agent might effectively use bidirectional communication with the operator to request further input from the operator to determine how to proceed in certain circumstances. In particular, in R-HATS the operator initially provides parameters (thresholds) that define how the agent will interact with the operator in making potentially risky decisions. When estimated risk for a particular course of action is below a threshold value, the automation can autonomously execute that course of action. If the risk is above this threshold the Agent prompts the operator for more input or guidance. When this happens, depending on the risk and threshold values, the system and operator may explore new options developed by the automation, or by the operator, with different risks and benefits. Through a series of prompts, the operator can work with the Agent in a back-and-forth manner to come to the best solution for a given situation.

\section{Use Operator-Directed Interfaces for Dynamic Task Delegation/Allocation}

Researchers have previously explored static schemes for the delegation of tasks between users and automation [10], [11]. Miller and Parasuraman [12] argue that the delegation of tasks between human operators and automation benefit HAT when performed in a dynamic manner resembling task delegation between humans. Operator-directed interfaces provide for the dynamic determination of levels of automation (LOA) [13] for tasks under the direction and ultimate authority of the operator [6]. The R-HATS Agent, described in Section 


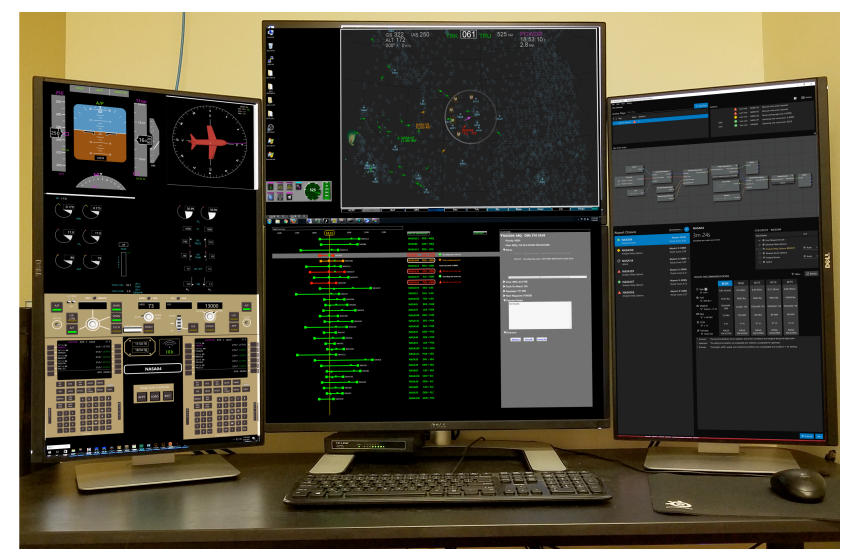

Fig. 1. The NASA RCO GCS. From left to right: aircraft instruments, traffic situation display (center-top), aircraft control list (center-bottom), and the R-HATS Agent.

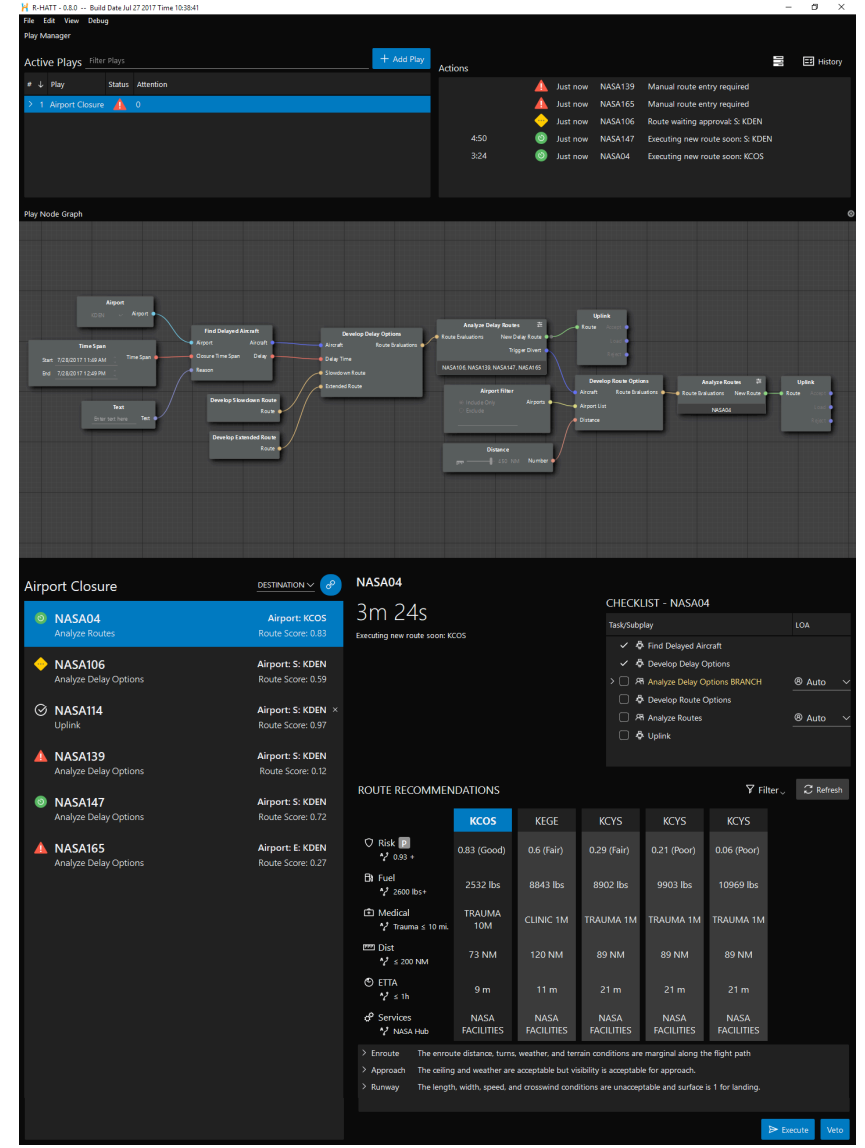

Fig. 2. Enlarged view of the R-HATS Agent while running an Airport Closure play for Denver International Airport.

III, employs a user-directed design facilitated through our application of plays [12], bi-directional communication, and an original, innovative strategy for dynamic, contextual determination of LOA.

\section{E. Coordinate Teamwork, Assets, and Tasks through the Usage of Plays}

Central to the aim of HAT is the optimal coordination of operators, assets, and their constituent tasks. Our approach to this coordination utilizes a human-autonomy integration architecture consisting of predefined "plays" for missions similar to those described by Miller \& Parasuraman [12]. In this context, a play is comprised of tasks or subplays with commonly understood goals together with a delegation (often dynamic) of roles and responsibilities to the automation and human operators. At the highest level the user can place a play into motion by "calling" it from a playlist. This concept is analogous to plays contained in the playbooks of professional sports teams, where the operator has supervisory control in a role akin to the team's coach. Calling a play consists of providing the specification of a desired goal via the play user interface. which then uses a shared vocabulary between operator and resources of how to achieve it.

Plays are hierarchical in composition, with other tasks and sub-plays nested within them. It is worth noting that the subplays can, in other contexts, be plays that the operator directly calls. So the design of a play often involves the selection and combining of sub-plays. Plays and sub-plays can also be modified or tailored prior to, or sometimes during, play execution. The possible paths to achieving the goal are adjusted as the situation evolves, either by the R-HATS Agent (e.g., through dynamic assignment of LOA) or through direct specification from the operator (e.g., changes to parameters determining this assignment of LOA). By utilizing the play concept, the operator's capabilities are enhanced by the ability to quickly place a coordinated plan in motion, monitor mission progress during play execution, and fine-tune mission elements dynamically as the play unfolds.

\section{R-HATS AGENT AND INTERFACE}

The HAT agent concepts described above can be supported by a variety of potential interfaces designed to meet the special needs of particular work domains. One such implementation, integrated into NASA's RCO GCS and developed as part of a collaborative effort between HATS, Inc., and NASA Ames Research Center is shown in Fig. 1. The GCS components in Fig. 1 consist of aircraft instruments for a selected aircraft (left monitor), a traffic situation display (TSD, center-top monitor), an aircraft control list (ACL, center-bottom monitor), and the R-HATS agent (right monitor). (For more information about the NASA RCO GCS, see [1], [4], and [14].) An enlarged image of the R-HATS agent is shown in Fig. 2.

In the pictured example, Denver International Airport (DEN) has been closed due to a thunderstorm and the RHATS agent is assisting six aircraft enroute to DEN. R-HATS considers contextual factors for the affected aircraft (e.g., risk, location, weather, fuel consumption, estimated delay times, medical facilities, and airline services) to generate and analyze options to either "absorb" the delay resulting from the closure enroute (e.g., by slowing down or modifying the route to DEN) or to divert to a suitable alternate airport. These contextual factors are considered by R-HATS against user defined thresholds for when R-HATS can autonomously 
decide to set an action in motion for a given aircraft, or for when it requires greater consideration from the operator.

The R-HATS agent interface (Fig. 2) consists of two principal components: the Play Manager and the Play Conductor. The Play Manager shows a list of actively running plays (top left) and current actions requiring further operator input (top right). Icons are displayed next to listed actions to indicate R-HATS' LOA determination given user-defined contextual factors for each aircraft. Below the Play Manager is the Play Conductor, itself consisting of a "node graph" (Fig. 2, center), aircraft list (Fig. 2, bottom-left), and a recommendation pane (Fig. 2, bottom-right). The node graph represents a high-level overview of the Aircraft Closure play as it unfolds in real time. Nodes correspond to inputs, tasks, and sub-plays that together define a play. Aircraft call signs are displayed below nodes to indicate their position in the course of the play. The aircraft list shows the aircraft involved in the currently selected play along with information regarding recommended actions icons representing their respective LOAs. To the right of this list is the recommendation pane, which provides further details (e.g., transparency information about a given diversion and the automation's reasoning behind suggesting it) about actions suggested by R-HATS for the aircraft selected in the list.

\section{CONCLUSION}

As an integrated component of the overall NASA RCO GCS, the R-HATS agent works together with suites of GCS automation to consider aircraft contextual factors against the operators preferences in determining who (i.e., agent or operator) will have ultimate responsibility for a given task, while preserving an operator's ultimate decision authority to override the agent. Here, we have detailed several tenets useful in the design of systems employing HAT and cursorily described an example implementation in the form of the RHATS Agent. Through the application of these tenets, HAT shows promise to alleviate operator workload while improving efficiency, performance, and safety.

\section{ACKNOWLEDGMENT}

The authors would like to thank Mr. Thomas Quinonez and Mr. Jonathan Luk for their help recreating the RCO GCS environment at HATS, Inc. Support for this work was provided by NASA Research Announcement Contract \#NNA15BDB6C, Mr. Robert J. Shively technical monitor.

\section{REFERENCES}

[1] S. V. Ligda, U. Fischer, K. Mosier, M. Matessa, V. Battiste, and W. W. Johnson, "Effectiveness of advanced collaboration tools on crew communication in reduced crew operations," in Eng. Psychology and Cognitive Ergonom.: $12^{\text {th }}$ Int. Conf., Los Angeles, CA, pp. 416-427.

[2] M. R. Endsley, "From here to autonomy: lessons learned from humanautomation research," Human Factors, vol. 59, no. 1, pp. 5-27, 2017.

[3] K. M. Feigh, and A. R. Pritchett, "Requirements for effective function allocation: a critical review," J. of Cognitive Eng. and Decision Making, vol. 8, no. 1, pp. 23-32, 2014.

[4] S. L. Brandt, J. Lachter, R. Russell, and R. J. Shively, "A humanautonomy teaming approach for a flight following task," in Advances in Neuroergonom. and Cognitive Eng. AHFE 2017. Advances in Intell. Syst. and Comput., vol. 586, C. Baldwin, Ed. Cham, Switzerland: Springer, 2018, pp. 12-22.

[5] J. Lachter, S. L. Brandt, G. Sadler, and R. J. Shively, "Beyond point design: general pattern to specific implementations," in Advances in Neuroergonom. and Cognitive Eng. AHFE 2017. Advances in Intell. Syst. and Comput., vol. 586, C. Baldwin, Ed. Cham, Switzerland: Springer, 2018, pp. 34-45.

[6] R. J. Shively et al., "Why human-autonomy teaming?," in Advances in Neuroergonom. and Cognitive Eng. AHFE 2017. Advances in Intell. Syst. and Comput., vol. 586, C. Baldwin, Ed. Cham, Switzerland: Springer, 2018, pp. 3-11.

[7] M. R. Endsley, "Toward a theory of situation awareness in dynamic systems," Human Factors, vol. 37, no. 1, pp.32-64, 1995.

[8] J. B. Lyons, "Being transparent about transparency: a model for humanrobot interaction," in 2013 Assoc. for the Advancement of Artificial Intell. Spring Symp., Palo Alto, CA, 2013, pp. 48-53.

[9] J. Y. Chen, K. Procci, M. Boyce, J. Wright, A. Garcia, and M. Barnes, "Situation awareness-based agent transparency," Army Research Lab., Aberdeen, MD, Rep. ARL-TR-6905, Apr. 2014.

[10] M. R. Endsley, and E. Kiris, "The out-of-the-loop performance problem and level of control in automation," Human Factors, vol. 37, no. 2, pp. 381-394, 1995.

[11] C. D. Wickens, A. Mayor, R. Parasuraman, and J. McGee, The future of air traffic control: human operators and automation. Washington, DC: Nat. Academy Press, 1998.

[12] C. A. Miller, and R. Parasuraman, "Designing for flexible interaction between humans and automation: delegation interfaces for supervisory control," Human Factors, vol. 49, no. 1, pp. 57-75, 2007.

[13] T. B. Sheridan, and W. L. Verplank, "Human and computer control of undersea teleoperators," Office of Naval Research, Arlington, VA, Rep. N00014-77-C-0256, Nov. 1978.

[14] G. Sadler et al., "Effects of transparency on pilot trust and agreement in the autonomous constrained flight planner," in Digital Avionics Syst. Conf. (DASC), 2016 IEEE/AIAA 35 , Sacramento, CA, 2016. doi: 10.1109/DASC.2016.7777998 